ARTÍCULO

\title{
Aprendizaje informal del profesorado: vinculación con el desarrollo profesional y organizacional
}

\author{
Joaquín Gairín Sallán' (D) \\ José Luís Muñoz Moreno' (D) \\ Patricia Silva García" iD \\ Cecilia Inés Suárez' iD
}

\section{RESUMEN}

El artículo analiza el aprendizaje informal del profesorado y su vinculación con el desarrollo profesional y organizacional. La revisión de la literatura detalla las implicaciones de estas para las organizaciones en general, y centros educativos en particular. Se destaca la importancia de la práctica reflexiva del profesorado, sus actitudes positivas ante el cambio y el trabajo colaborativo. La promoción del aprendizaje informal queda apoyada en factores individuales, grupales y organizacionales, base para una propuesta pedagógica para fomentar el aprendizaje informal en los centros educativos. Como conclusión, se apuntan posibilidades y limitaciones del aprendizaje informal para las organizaciones que aspiran a su consideración como elemento para la mejora profesional y organizacional.

PALABRAS CLAVE

aprendizaje informal; profesorado; desarrollo organizacional; desarrollo profesional. 


\title{
TEACHER'S INFORMAL LEARNING: RELATION WITH PROFESSIONAL AND ORGANIZATIONAL DEVELOPMENT
}

\begin{abstract}
The article analyzes the informal learning of the teaching staff and its relationship with the professional and organizational development. The literature review details the implications of these for organizations in general, and educational centers in particular. The importance of reflective practice of teachers, their positive attitudes toward change and collaborative work are also highlighted. Individual, group and organizational factors support the promotion of informal learning, as the basis for a pedagogical proposal to encourage informal learning in schools. In conclusion, possibilities and limitations of informal learning are emphasized, especially for organizations that aspire to their consideration as an element for professional and organizational improvement.
\end{abstract}

KEYWORDS

informal learning; teachers; organizational development; professional development.

\section{APRENDIZAGEM INFORMAL DE PROFESSORES: RELAÇÃO COM O DESENVOLVIMENTO PROFISSIONAL E ORGANIZACIONAL}

\section{RESUMO}

$\mathrm{O}$ artigo analisa a aprendizagem informal dos professores e a sua relação com o seu desenvolvimento profissional e organizacional. A revisão da literatura evidencia as implicações da aprendizagem informal para as organizações em geral e para as escolas em particular. Destaca-se a importância da prática reflexiva dos professores, das suas atitudes positivas face à mudança e do trabalho colaborativo. A promoção da aprendizagem informal apoia-se em fatores individuais, grupais e organizacionais que são base para uma proposta pedagógica de fomento da aprendizagem informal nas escolas. Como conclusão, apontam-se as possibilidades e as limitações da aprendizagem informal para as organizações que procuram considerá-la como um fator para a melhoria profissional e organizacional.

\section{PALAVRAS-CHAVE}

aprendizagem informal; corpo docente; desenvolvimento organizacional; desenvolvimento profissional. 


\section{INTRODUCCIÓN}

La creación y difusión del conocimiento y las facilidades actuales para conocerlo y apropiarse de él están modificando el sentido y contenido de las organizaciones formativas y no formativas. Actualmente, las personas pueden adquirir conocimientos y desarrollar destrezas por su cuenta y al margen de los sistemas reglados de formación. Los aprendizajes informales son cada vez más abundantes y dichas organizaciones se plantean retos con relación a su identificación, reconocimiento e institucionalización.

El aprendizaje informal se produce lejos de las acciones formativas organizadas y se sitúa entre los límites de la información y la formación (Martínez y Muñoz, 2018). A priori, carece de procesos estructurados de evaluación y, la mayoría de las veces en que tiene lugar, tampoco cuenta con soportes pedagógicos. Las formas más habituales de aprendizaje de las personas incluyen el aprendizaje informal (Cross, 2006): conversaciones, reflexiones, narraciones de historias, explicaciones de vivencias y experiencias, intercambios de conocimiento con otros, etc. Y se genera mediante procesos educativamente indiferenciados e inespecíficos (Trilla, 1987), y también puede darse en situaciones que en sí mismas no tienen un claro propósito educativo.

Aunque el contexto educativo es, por su naturaleza y sistema de funcionamiento, un entorno donde también tiene cabida el aprendizaje informal, no siempre se ha considerado para identificar sus posibilidades y limitaciones. El sistema reglado de los estudios, el peso de la academia, una fuerte tradición de formación reproductiva y el miedo a lo desconocido pueden explicar la falta de consideración de un sistema de aprendizaje que supone, en muchos casos, más del $70 \%$ de lo que saben las personas.

Pero lo que nos preocupa no es tanto analizar en sí mismo el aprendizaje informal sino la manera cómo los centros educativos pueden incorporar ese bagaje al funcionamiento institucional. Particularmente, se plantea la necesidad de convertir el aprendizaje informal del profesorado en un instrumento para su desarrollo profesional y, a la vez, para el desarrollo organizacional de los centros educativos. De hecho, el objetivo principal de la contribución es analizar las relaciones entre el aprendizaje informal del profesorado y su vinculación con el desarrollo profesional y organizacional. Parte, al respecto, de una revisión de la literatura específica y de analizar las implicaciones que tiene para los centros educativos (Figura 1). Revisadas las aportaciones teóricas más relevantes, se derivan consecuencias para el contexto educativo, a la vez que se identifican las principales posibilidades y limitaciones de una determinada propuesta pedagógica.

El desafío actual no está en convencer de las bondades del aprendizaje informal para los centros educativos, ni tan si quiera en señalar herramientas que lo faciliten y amplifiquen. Aunque el aprendizaje informal pudiera estar siendo ignorado en muchas organizaciones, bien por falta de conocimiento o porque los entornos no son apropiados para ello, lo que nos interesa es reflexionar sobre como las organizaciones enfocan cuestiones como esta: ¿cómo los centros educativos pueden favorecer el aprendizaje informal del profesorado? Seguramente, el 


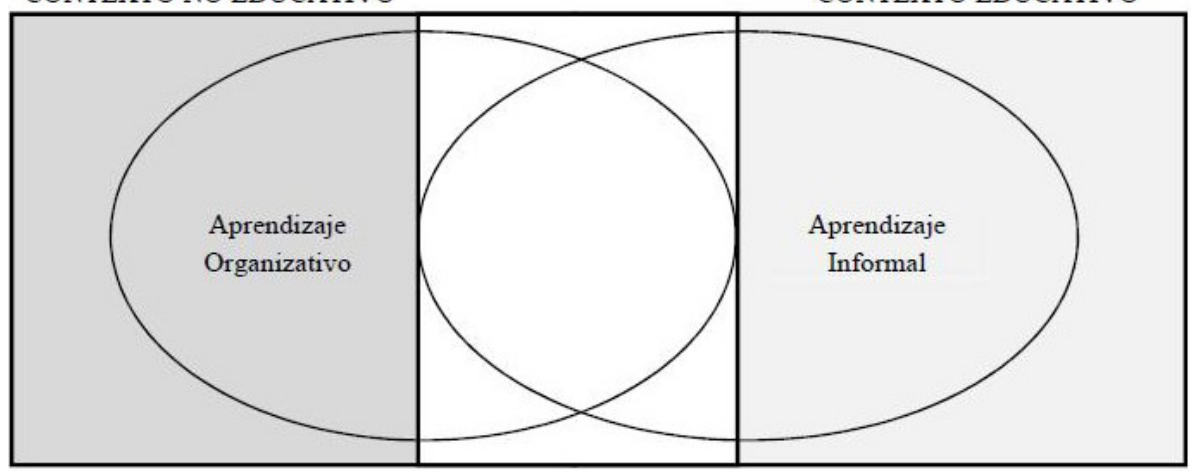

Figura 1 - Tópicos para el análisis.

Fuente: Base de datos de investigación.

Elaboración propia.

aprendizaje de las organizaciones trasciende la suma de aprendizajes individuales (Bolívar, 2000), y esto se relaciona con que las personas confronten problemáticas y articulen alternativas, lo que llevaría a unas organizaciones a aprender y crecer más que otras (Pearn, Roderick y Mulrroney, 1995).

El foco se fija en la relación entre el aprendizaje individual, el aprendizaje organizativo y la gestión del conocimiento colectivo, de acuerdo con aportaciones anteriores (Gairín, 2016, 2015). El desarrollo de estrategias de aprendizaje organizativo y de gestión del conocimiento colectivo permiten a las organizaciones adaptarse a los cambios que la realidad impone, mejorando el rendimiento de los trabajadores y de la organización.

La aportación se conecta al estudio Análisis de los procesos de aprendizaje organizativo e informal en los centros educativos. Validación de propuestas tecnológicas para el desarrollo institucional y profesional docente y enfatiza como ensayo en la relación entre el aprendizaje individual y el aprendizaje organizativo, que se puede hacer realidad a partir de planes sistemáticos de intervención que emplean estrategias e instrumentos adecuados para la transformación deseada por los centros educativos.

\section{A PROPÓSITO DEL APRENDIZAJE INFORMAL}

Es definido como aquel que se produce como resultado de situaciones no estructuradas pedagógicamente; esto es, en las que no existe un currículum, un objetivo o intencionalidad pedagógica, o roles claramente delimitados entre quienes aprenden y quienes enseñan y, por consiguiente, no es sistemático ni intencional.

El aprendizaje informal contribuye al desarrollo profesional del profesorado por medio del conocimiento acumulado y compartido con los demás desde una perspectiva colaborativa (Martínez y Muñoz, 2018). De hecho, la colaboración es clave en el desarrollo profesional docente (Martínez, 2017; Trillo et al., 2017) y relevante en los procesos formativos y de innovación educativa (Escudero, Cutanda y Trillo, 2017). Propuestas como el modelo 70/20/10 facilitan que así sea (Jennings, 2012; Martínez, Muñoz y Falivene, 2017) y, como 
dicen Scott y Ferguson (2016), se trata de un 70\% de aprendizaje experiencial (resolución de problemas, tareas retadoras, reflexión deliberada, etc.), un 20\% de aprendizaje social (peer coaching, dar y recibir retroalimentación, "mentoring", etc.) y un $10 \%$ de aprendizaje formal (congresos, cursos, seminarios, talleres, etc.). También lo hacen otras estrategias mediadas por las tecnologías (González y Gutiérrez, 2013), como las comunidades de práctica en contextos de trabajo que apoyan un aprendizaje colectivo a favor de un desarrollo profesional efectivo (Bolívar, 2014).

La experiencia "Horizont 2020" (Aragay, 2017; Aragay et al., 2015) ha incitado en el contexto español pedagogías invisibles, sin clasificaciones curriculares fuertes, sin fragmentaciones de los conocimientos en materias y sin rigidez en las relaciones de poder en el aula (Martínez, 2015). Por eso, la apertura a otros enfoques desde una concepción de aprendizaje híbrido proporciona auténticas oportunidades para el desarrollo profesional del profesorado (Arets, 2017).

Muchos elementos de la definición de aprendizaje informal sirven para caracterizar esta modalidad, pudiendo destaca (Fernández, 2014):

- Su asociación con las actividades de la vida cotidiana (en los ámbitos de la familia, el trabajo y el ocio, por ejemplo).

- Estar controlado por la propia persona, al no obedecer a planificaciones institucionales o personales dedicadas a la formación.

- No contar con un currículum formal (no está estructurado en objetivos didácticos, soporte y duración).

- No conducir a una certificación.

El aprendizaje informal es un eje fundamental para el aprendizaje organizativo, aunque cabe preguntarse por qué y cómo debería gestionarse en los centros educativos considerando que es una actividad que, en principio, no puede ser controlada o manipulada por agentes externos. Al corresponder con un aprendizaje experiencial puede seguir, de acuerdo con Kolb (1984), este ciclo: aprender experimentando (experiencia concreta), aprender procesando (observación reflexiva), aprender generalizando (conceptualización abstracta) y aprender haciendo (experimentación activa).

No obstante, si se considera la organización como una organización que aprende y la asociamos a que desarrolla internamente: "la capacidad de adaptarse o responder rápidamente y de manera innovadora, cuando remueve las barreras para el aprendizaje" (Marsick y Watkins, 1990), es posible comprender el interés de las organizaciones por incidir en el aprendizaje informal a partir de dos tipos de actividades (Barrera-Corominas, 2015):

1. Desarrollo de procesos de visualización, comunicación y conexiones entre las personas que las conforman. Por ejemplo, a partir de la diversificación de los canales de comunicación y su uso virtual o presencial.

2. Promoción de herramientas y recursos para la identificación y evaluación de los aprendizajes y conocimientos que las personas adquieren fuera del contexto formativo, y que son útiles a la propia organización. 
Para que se produzca el aprendizaje organizativo cabe esperar que la organización haya desarrollado ciertas estrategias y procedimientos, con la finalidad de que cada miembro de ella cuente con el conocimiento necesario para llevar adelante sus tareas profesionales (Martínez-Aldanondo, 2010).

Crossan, Lane y Withe (1999) ya señalaron que se trata de un proceso dinámico y en constante evolución. Estimaban un modelo de aprendizaje organizacional basado en cuatro procesos interrelacionados que ocurren en tres niveles. En este modelo, los procesos de intuición, interpretación, integración e institucionalización se desarrollan a través de los niveles individual, grupal y organizacional, en una secuencia y ordenación que produce diferentes resultados. Sin embargo, no todos los procesos se dan en todos los niveles: el nivel individual comprende los procesos de intuición e interpretación, mientras que los niveles grupal y organizacional se corresponden con los procesos de integración e institucionalización, respectivamente.

Por su parte, para Martínez-Aldanondo (2016) son tres los hábitos que deben consolidarse en las organizaciones si se quiere favorecer su aprendizaje: reflexionar, sistematizar y compartir. La instauración de estos hábitos, que deben tener continuidad y un tiempo en el marco de las actividades rutinarias de la organización, permiten aprender de las acciones que los profesionales desarrollan cada día y que pasan desapercibidas como oportunidades de aprendizaje organizacional.

Esta opción se sustenta en incorporar los hábitos de preguntarse, por ejemplo, qué salió bien, por qué y cómo mantenerlo en el futuro (reflexionar); por seleccionar la información obtenida y documentarla para poder recuperarla tantas veces como sea necesario (sistematizar); y por intercambiar el conocimiento generado en un ejercicio de colaboración constante entre profesionales y organizaciones. Pero la condición fundamental radica en concebir el aprendizaje dentro de la organización como una inversión que está justificada, entre otras cuestiones porque mejora los procesos internos y ayuda a no repetir errores, explicándose así el que se dedique tiempo, espacio y recursos de manera sistemática y rutinaria.

Cross (2010) sugiere algunas orientaciones para mejorar la calidad del aprendizaje informal en las organizaciones, entre las que se encuentran: legitimar el aprendizaje informal en las organizaciones; reconocer que el aprendizaje es el trabajo y el trabajo es el aprendizaje; promover la confianza, la colaboración y la conexión con los demás; eliminar los obstáculos a la conversación y la experimentación; optimizar la ecología de aprendizaje de la organización; ayudar a los trabajadores a aprender a aprender; y buscar la innovación. También hay que considerar y prevenir algunos inconvenientes derivados del contexto, ya que, especialmente, encaja con las necesidades de la situación donde se produce (Machado y Pohl, 2004) y, además, tiene un carácter de incontrolado y dinámico que puede llegar a generar incertidumbres.

De todos modos, no solo conviene promover e intercambiar el aprendizaje informal sino, sobre todo y una vez cualificado por su utilidad para la organización, generar procesos de formalización e institucionalización que favorezcan integrarlo en la mejora de la organización y, por supuesto, en el desarrollo profesional. Aunque esto pudiera parecer un cierto oxímoron, la principal pretensión enlaza con que el aprendizaje informal sea una realidad valiosa tanto para las personas como para las organizaciones. 


\section{LOS FACTORES ASOCIADOS AL APRENDIZAJE INFORMAL EN LAS ORGANIZACIONES}

Los factores que pueden participar de la promoción del aprendizaje informal en el ámbito organizacional son múltiples y diversos. Varias son las aportaciones que dan cuenta de estos (AECA, 2011; Baigorri, Martínez y Monterrubio, 2006; Carmelo, García y Sousa, 2010; Cuadros, 2015; Galindo, 2013; LLP, 2010; Orozco, 2005; Retortillo, 2011; Riera, 2013; Sangrà y Wheeler, 2013; Serrano y Dalmau, 2010; UNESCO, 2012) y que los clasifican en tres niveles: individuales, grupales y organizacionales. Su caracterización exhaustiva toma como referente principal los trabajos clásicos de Watkins y Marsick (1993) sobre aprendizaje informal y los de Crossan, Lane y White (1999) sobre aprendizaje organizacional. Una síntesis de los factores considerados se analiza a continuación.

\section{FACTORES INDIVIDUALES}

Las organizaciones promueven la utilización del aprendizaje informal desde factores individuales tales como:

- El aprendizaje por error, la identificación de las habilidades necesarias para la realización de las tareas profesionales o el apoyo mutuo en el aprendizaje.

- La obtención de recursos para facilitar el aprendizaje, el tiempo disponible para aprender, la consideración de problemas como oportunidades de aprendizaje y la retroalimentación colaborativa (abierta y honesta).

- Otros: la escucha sobre dicha retroalimentación, la petición de explicaciones sin preocupar el escalafón profesional, la emisión y la recepción de opiniones, el trato respetuoso o la construcción de relaciones de confianza.

El aprendizaje informal exige de una cierta actitud individual favorable y comprometida con el propio aprendizaje. Esto supone aprender haciendo y resolviendo individualmente las dificultades. Los errores representan acciones equivocadas, pero, al mismo tiempo, se convierten en una vía potente de aprendizaje debido a que las personas frecuentemente no se atreven a cometer errores (Cross, 2007; Fernández, 2014; Marsick y Watkins, 1990; Prieto, 2005; Senge, 1992).

Si partimos de la concepción de que el aprendizaje informal en el puesto de trabajo se produce cuando las personas no tienen las competencias necesarias para resolver las problemáticas que aparecen en el desarrollo de su actividad laboral (Fernández, 2014; Marsick y Watkins, 1990), no cabe duda de que todo profesional precisa identificar qué competencias le son requeridas para consolidarlas, aplicarlas y mejorarlas (Eraut, 2008). Las organizaciones deberían facilitar, al respecto, la identificación de competencias, junto con las experiencias laborales, facilitando su intercambio y favoreciendo así el apoyo mutuo en el aprendizaje. Cabe recordar aquí, con Eraut (2004), que la retroalimentación es un factor con un elevado impacto sobre el aprendizaje. 
Compartimos con Nicol (2010) los beneficios de la retroalimentación, entre otros:

- La ayuda para aclarar qué significa una buena práctica.

- La motivación para dedicar tiempo y esfuerzo a tareas de aprendizaje individual y grupal.

- Proporcionar información de alta calidad que ayuda a las personas en su aprendizaje individual.

- Proveer de oportunidades para minimizar cualquier diferencia entre el aprendizaje actual y el deseado.

- Favorecer un impacto positivo sobre el aprendizaje organizativo.

Igualmente, posibilita la interacción y el diálogo entre pares, facilita la reflexión sobre el aprendizaje adquirido, implica a las personas en la toma de decisiones sobre su propio desarrollo, apoya la creación de comunidades de práctica, estimula la autoestima y la motivación personal y grupal y proporciona información a responsables institucionales que la pueden usar para la mejora del aprendizaje. La retroalimentación tiene que ser ágil y destacar aquello que se hace bien, aquello que hay que mantener y aquello que hay que repensar. No solo debe informar de los errores sino también de los motivos por los que se cometen y ofrecer sugerencias para superarlos y probar otras formas de resolución.

Por eso, los profesionales pueden adoptar roles tan diversos como mediadores y facilitadores de aprendizaje y conocimiento, formadores, mentores, tutores, dinamizadores, autores, consultores, coachs, editores, correctores, maquetadores o creadores de conocimiento y editores de información.

En el fondo, implica ubicar a las personas en el centro de los procesos de aprendizaje a partir de la autorreflexión, la automotivación y la autoeficacia, para seguir aprendiendo desde la confianza y buscando aprendizajes compartidos que puedan gestarse desde el conocimiento propio y desde la experiencia profesional. Los principios que inspiran estos factores permanecen conectados a la igualdad de oportunidades, la equidad y la inclusión dentro de la lógica del aprendizaje a lo largo y ancho de toda la vida y de la formación permanente.

\section{FACTORES GRUPALES}

Estos factores se relacionan con la libertad de modificación de objetivos, el trato como iguales, la focalización en tareas y formas, la revisión de planteamientos, las recompensas por logros y la actuación de acuerdo con recomendaciones.

Su variedad apoya el que los factores grupales promuevan el aprendizaje informal logrando, por un lado, modificar los objetivos cuando resulta necesario y otorgando, por tanto, autonomía al equipo; por otro lado, desarrollar procesos de socialización mediante la participación en redes, sistemas de creación y gestión del conocimiento y otros recursos (contenidos virtuales, materiales, manuales, fichas, etc.). Pero compartir informaciones, experiencias o las mismas opiniones sólo tiene éxito cuando se instala una cultura del compartir basada en contribuciones y avances colectivos. Es en ese momento cuando la cultura colaborativa se distingue por el 
trato como iguales, la comunicación, el diálogo, el contraste de ideas, el trabajo en equipo y el apoyo mutuo.

La interacción entre los profesionales debe servir, sin duda, para construir inteligencia colectiva y un sistema de relaciones que haga de la interdisciplinariedad un valor realmente en alza. A menudo, el establecimiento de una óptima infraestructura de aprendizaje pasa por las recompensas de aprendizaje apoyadas en sistemas de recompensas grupales y en incentivos de aprendizaje, pudiéndose traducir también en recompensas para sugerir mejoras, por el establecimiento de una infraestructura de creación y gestión del conocimiento o por la configuración de un sistema de apoyo (Marsick y Watkins, 1990).

Las funciones de las personas encargadas de promover y gestionar el aprendizaje, coincidiendo con Sanz (2002), son destacables para identificar temas importantes, planificar y facilitar las actividades a desarrollar, potenciar el desarrollo profesional de las personas, gestionar la frontera entre el aprendizaje informal y la organización formal, ayudar a construir la práctica con el conocimiento base, valorar la salud organizativa y evaluar las contribuciones que se hacen.

El rol de estas personas, dinamizadoras y coordinadoras de aprendizaje grupal, consiste fundamentalmente en conectar informalmente a las personas superando los límites entre las unidades organizacionales, aprovechando la experiencia adquirida y el aprendizaje individual, así como las herramientas y las estrategias de aprendizaje para progresar conjunta y colectivamente.

\section{FACTORES ORGANIZACIONALES}

Sería el caso de:

- La comunicación bidireccional.

- La obtención rápida y fácil de información.

- La posesión de bases de datos actualizadas sobre habilidades y competencias.

- La medición de desajustes en el desempeño esperado y auténtico.

- La difusión de las lecciones aprendidas.

- La medición de resultados del tiempo y los recursos de formación.

- La toma de iniciativas.

- La oportunidad de trabajar en aquello que se quiere.

- La definición colaborativa de líneas estratégicas.

- El control sobre los recursos.

- La asunción de riesgos calculados.

- Los grupos de trabajo.

- La conciliación laboral y familiar.

- El pensamiento global.

- La expresión de opiniones.

- El impacto de las decisiones en la moral.

- El trabajo conjunto de toda la comunidad educativa.

- La búsqueda compartida de soluciones a los problemas.

- El compartir experiencias. 
- La disposición de tecnología para colaborar.

- El apoyo en la participación de actividades formativas.

- La actuación de mentores y formadores.

- La búsqueda continua de formas de aprender.

- La coherencia entre acciones y valores.

Los factores organizacionales que invitan a la generación del aprendizaje informal tienen que ver, en gran medida, con el clima y la cultura de las organizaciones, con sus estructuras organizativas y sistemas de apoyo, así como con un particular rol de los responsables institucionales en la línea de prestar ayuda, dar confianza, cumplir con los acuerdos, actuar con el sentido de la justicia y aspirar al empoderamiento. Se ubican así en la perspectiva de la organización que aprende y autocualificante (Argyris y Schön, 1978; Aubrey y Cohen, 1995; Martínez Marín, 2010).

Los procesos de toma de decisiones inciden notablemente en la promoción del aprendizaje informal, especialmente cuando hay una menor relación jerárquica en las organizaciones y cuando se dispone de autonomía para la gestión. Otros factores organizacionales tienen mucho que ver con la gestión de las personas y los recursos humanos. Por ejemplo, la motivación a los profesionales, el ofrecimiento de oportunidades para todos, la proximidad de roles, la delimitación de competencias profesionales o unos equipos de trabajo bien estructurados.

Resulta clave, además, la configuración de entornos predispuestos para crear y compartir el conocimiento en unos escenarios integradores de las tecnologías, impregnados de flexibilidad comunicativa y con unas prácticas contextualizadas de (in)formación pública. Las tecnologías son imprescindibles en la instauración de nuevas vías de aprendizaje y de culturas colaborativas en las organizaciones (Cobo y Moravec, 2011). Concretamente, las redes (personales, sociales, corporativas y de contactos virtuales), las comunidades de práctica y los ambientes personales de aprendizaje, son espacios virtuales muy propicios para el aprendizaje informal en el seno de las organizaciones.

La aspiración perseguida enlaza con la conexión entre el aprendizaje informal y el aprendizaje organizacional y, en coherencia, con lograr que las organizaciones puedan mejorar y desarrollarse en los distintos ámbitos organizativo, democrático, social, profesional y de extensión/internacionalización como oportunidad para ampliar sus perspectivas y cometidos.

Las organizaciones con un cierto interés en promover el aprendizaje informal tienen que reforzar su compromiso que, de acuerdo con López y Marcelo (2003), se realiza a través de la visualización (proporcionando herramientas e instrumentos para unas comunicaciones más efectivas y ágiles), las conversaciones (físicas y/o virtuales para el aprendizaje, el descubrimiento, el intercambio de conocimiento, etc.) y las conexiones (por distintos canales y siendo comprensibles, analizadas, apoyadas y como facilitadoras de aprendizaje individual y colectivo).

Algunas actuaciones que ayudan en la consecución de la meta fijada son: formular colaborativamente objetivos claros y precisos sobre lo que se pretende 
lograr, concretar roles y funciones de los agentes participantes, así como los mecanismos de evaluación y seguimiento, la valoración de costes y recursos necesarios, las actividades que se quieren implementar en coherencia con los objetivos fijados y la metodología y las acciones a efectuar, globales y específicas, desde criterios de flexibilidad y adaptabilidad.

La existencia en las organizaciones de una cultura institucional sostenida sobre la base de la colaboración para crear y compartir conocimiento y apoyar el aprendizaje informal es significativa (González-Isasi et al., 2013). La colaboración debe hacer posible mantener las sinergias institucionales y profesionales para desempeñar políticas eficaces y eficientes ante las necesidades y los cambios que puedan acompañar a la promoción del aprendizaje informal. Supone ello colaborar para compartir propósitos e implicarse en el logro de los objetivos y en la realización de las tareas correspondientes, garantizando la libertad de acción, la igualdad de trato, la lealtad, el compromiso, el respecto a la diferencia y la complementariedad desde la apertura y la solidaridad.

Pero la colaboración, que es un elemento sustancial para la innovación (Krichesky y Murillo, 2018; Ramírez y García, 2018), difícilmente tendrá lugar sin confianza, tanto entre los profesionales como en las organizaciones, para encarar el reto del aprendizaje informal y la creación y construcción de conocimiento colectivo. La edificación de esta imperiosa confianza puede originarse desde la realización de cosas en común y el intercambio de prácticas (Brown y Duguid, 2000), hasta la muestra de claridad con lo que se espera, pasando por el compromiso desde uno mismo y con los demás, el cumplimiento de lo prometido, la toma de consciencia de lo que se hace y sus repercusiones, la coherencia entre lo que se dice y lo que se hace, la consistencia en las formas de proceder y la intervención desde unos principios determinados (Gasalla y Navarro, 2008).

Avanzar en la dirección de los tres tipos de factores analizados es progresar en el sentido de las ventajas que tiene el aprendizaje informal y que guardan una enorme conexión con aprovechar la utilización colectiva del conocimiento, poniéndolo al servicio de los procesos de mejora y de la innovación. También beneficia a las personas cuando se certifica, acredita, reconoce y valida, puesto que visibiliza competencias ocultas y valoriza otras adquiridas por medio de la experiencia profesional. Por eso, conviene enfatizar tanto en los procesos como en los resultados de aprendizaje, propiciando intercambios en sistemas abiertos, flexibles e inclusivos; también, conectando lo formal y lo no formal para satisfacer las necesidades formativas que el aprendizaje informal trae consigo y pretende incorporar.

$\mathrm{El}$ aprendizaje informal representa aproximadamente un $80 \%$ del aprendizaje que se genera dentro de las organizaciones (Serrano y Dalmau, 2010) y, por eso, es nodal identificar los principios que contribuyen a su impulso. En nuestro caso, los comprendemos como el conjunto de valores, creencias y criterios que regulan y orientan la vida organizacional desde la perspectiva del aprendizaje (Cuadro 1).

Sea como fuere se aprende con entusiasmo (Mejía, 2005) y por el entusiasmo de otros, si consideramos que el aprendizaje se construye a partir de compartir experiencias con otros y porque el intercambio de ideas y dudas es parte sustancial en el logro de los propósitos comunes. La forma de involucrarse es voluntaria, con- 
Cuadro 1 - Valores y principios para el fomento del aprendizaje informal.

\begin{tabular}{|c|c|}
\hline Participación & $\begin{array}{l}\text { No existe una intención prescriptiva de enseñanza, sino que más bien } \\
\text { el proceso de aprendizaje es espontáneo o autodirigido. Para Mejía } \\
\text { (2005), es el aprendizaje adquirido por medio de la experiencia y se } \\
\text { asocia frecuentemente a la idea de aprendizaje en la práctica. El estudio } \\
\text { de Sánchez-Valle (2003) viene a reforzar la importancia de materializar } \\
\text { procesos de escucha activa para favorecer relaciones sostenidas en el } \\
\text { diálogo participativo. }\end{array}$ \\
\hline Motivación & $\begin{array}{l}\text { Ayuda al aprendizaje individual porque se produce cuando la persona } \\
\text { que aprende sigue una ruta elegida y controlada. Tiene, pues, mucho de } \\
\text { voluntad e interés propio y es "un aprendizaje de libre elección” (Falk y } \\
\text { Dierking, 1995). }\end{array}$ \\
\hline $\begin{array}{l}\text { Escenarios de } \\
\text { aprendizaje }\end{array}$ & $\begin{array}{l}\text { Provocan situaciones y contextos específicos dónde ocurre el aprendizaje y } \\
\text { la posibilidad de que las personas puedan acceder a la información desde } \\
\text { la curiosidad en un ambiente propicio (Salinas, 2003). }\end{array}$ \\
\hline Clima relacional & $\begin{array}{l}\text { Permite avanzar hacia la institucionalización en base al respeto a los } \\
\text { derechos de las personas y el uso del diálogo como eje de aprendizaje } \\
\text { colaborativo. Es un instrumento eficaz para la formación en la } \\
\text { convivencia. }\end{array}$ \\
\hline $\begin{array}{l}\text { Retroalimentación } \\
\text { prospectiva }\end{array}$ & $\begin{array}{l}\text { Estimula que las aportaciones de las personas tengan implicaciones } \\
\text { en tareas inmediatas y mediatas. Para Padilla y Gil (2008) supone que } \\
\text { las acciones implementadas sean realizadas mejor en el futuro y ante } \\
\text { actividades similares. La retroalimentación, según recomendaciones } \\
\text { de JISC (2010), debe basarse en evidencias múltiples, tareas reales y } \\
\text { ambientes profesionales interactivos y proactivos. }\end{array}$ \\
\hline
\end{tabular}

Fuente: Base de datos de investigación.

Elaboración propia.

tándose con una motivación inherente al interés por aprender. Y en estos propósitos comunes, las personas controlan el grado de implicación, profundidad y dedicación temporal para la inversión en el aprendizaje.

\section{LA PROMOCIÓN DEL APRENDIZAJE INFORMAL EN LOS CENTROS EDUCATIVOS}

Si se asume que siempre existe aprendizaje informal en los centros educativos, parece pertinente plantearse si la naturaleza específica de estas organizaciones remarca de alguna manera la presencia de ciertos factores vinculados a este aprendizaje y que se deberían de considerar.

La consideración de los factores individuales enlaza con el discurso del profesorado como profesional reflexivo y con la existencia de una actitud positiva ante el cambio. Por una parte, analizar las prácticas profesionales y aprender de los errores aleja a los profesionales de un modo de hacer rutinario que utilizan modelos repetitivos de situaciones anteriores sin considerar la naturaleza contextual y siempre variable de los procesos de enseñanza y aprendizaje. Por otra parte, la 
existencia de una positiva actitud hacia el cambio entiende la doble necesidad de buscar soluciones a nuevos problemas y de superar el riesgo a lo desconocido como la base de la experimentación y la mejora.

Dewey ya definía la acción reflexiva como la acción que supone la consideración activa, persistente y cuidadosa de toda creencia a la luz de los fundamentos que la sostienen y de las consecuencias a las que conduce. Identificaba tres actitudes vinculadas a esta acción (Dewey, 2010):

1. Apertura intelectual o deseo activo de considerar más de un punto de vista.

2. Responsabilidad o consideración de las consecuencias a las que conduce la acción.

3. Sinceridad vinculada a que los profesionales asuman el protagonismo de su propio aprendizaje.

$\mathrm{Su}$ influencia es notable y los desarrollos posteriores y actuales (Comas y Piqué, 2010; Domingo y Gómez, 2014; Domínguez, 2002; Perrenoud, 2004; Quinto y Fenero, 2016; Roget, 2013; entre otros) inciden en procedimientos y estrategias que ayudan al profesorado a hacer realidad la práctica reflexiva (Schön, 1998).

Tomar en cuenta los factores grupales nos aproxima al trabajo colaborativo del profesorado, que comparte objetivos y establece relaciones horizontales que favorecen los intercambios y ayudan a configurar una inteligencia colectiva. La actividad de los líderes y coordinadores de los grupos docentes y el trabajo sistemático que puedan plantear en promover tanto el aprendizaje informal como su registro y formalización es capital en este punto. Escarabajal (2015), García-Valcárcel (2015), Lavie (2011) y López-Rodríguez (2002), entre otros, han insistido en los efectos positivos de la colaboración y en las condiciones que la hacen posible.

Los factores organizacionales en el marco de los centros educativos son primordiales en la medida en que los procesos normativos condicionan, $\mathrm{y}$ mucho, su vida y determinan en gran parte el clima y la cultura de estas instituciones. A este nivel, resulta sustancial considerar la existencia y el buen funcionamiento de los equipos de profesorado. Las estructuras de coordinación vertical (departamentos y semanarios), de coordinación horizontal (equipos de curso) o mixtas (equipos de ciclo y departamentos específicos como el de orientación) representan el epicentro donde se puede dar el trabajo colaborativo en los centros educativos. Su efectividad está en función de la existencia de planes de trabajo focalizados en temáticas concretas y de estrategias operativas facilitadoras de la concreción de los compromisos del profesorado (Armengol, 2002; Bonals, 2013; López, 2007; Olmos, 2012).

A partir de los tres ámbitos de factores, y que inciden en el aprendizaje informal, se puede afirmar que la producción y desarrollo de este tipo de aprendizaje, ligado al desarrollo de la organización, se ubica en los equipos de profesorado de los centros educativos siempre que promuevan el análisis de la práctica, la reflexión sobre sus causas y consecuencias y la adopción y verificación de compromisos dirigidos al cambio.

Este contexto puede garantizar los criterios de Serrano y Dalmau (2010) para el desarrollo del aprendizaje informal si consideramos que, en los equipos de 
profesorado, es posible encontrar participación, motivación, escenarios de aprendizaje, clima relacional y retroalimentación prospectiva. También es cierto que, muchas veces, no se dan estos elementos en la cotidianidad, lo que puede explicar su escasa efectividad. Sucede cuando no hay una planificación previa de las reuniones, los procesos de trabajo no obedecen a una dinámica efectiva o existe un clima de trabajo enrarecido por cuestiones personales, laborales o sociales que impiden concentrarse en el análisis de la práctica educativa.

Una propuesta pedagógica para el desarrollo del aprendizaje informal debería concentrarse en todas estas consideraciones y fijarse en los modos operativos que permiten conseguir los avances profesionales e institucionales deseados. La Figura 2 sintetiza lo que podría ser una propuesta de trabajo contextualizada para los centros educativos de educación infantil y primaria y de educación secundaria. Se apoya en los marcos teóricos presentados pero también en realizaciones prácticas como puedan ser los estudios sobre prácticas educativas basadas en evidencias, ${ }^{1}$ la incorporación a las redes de intercambio profesional de espacios para el debate colectivo y la generación de documentos compartidos, ${ }^{2}$ la extensión

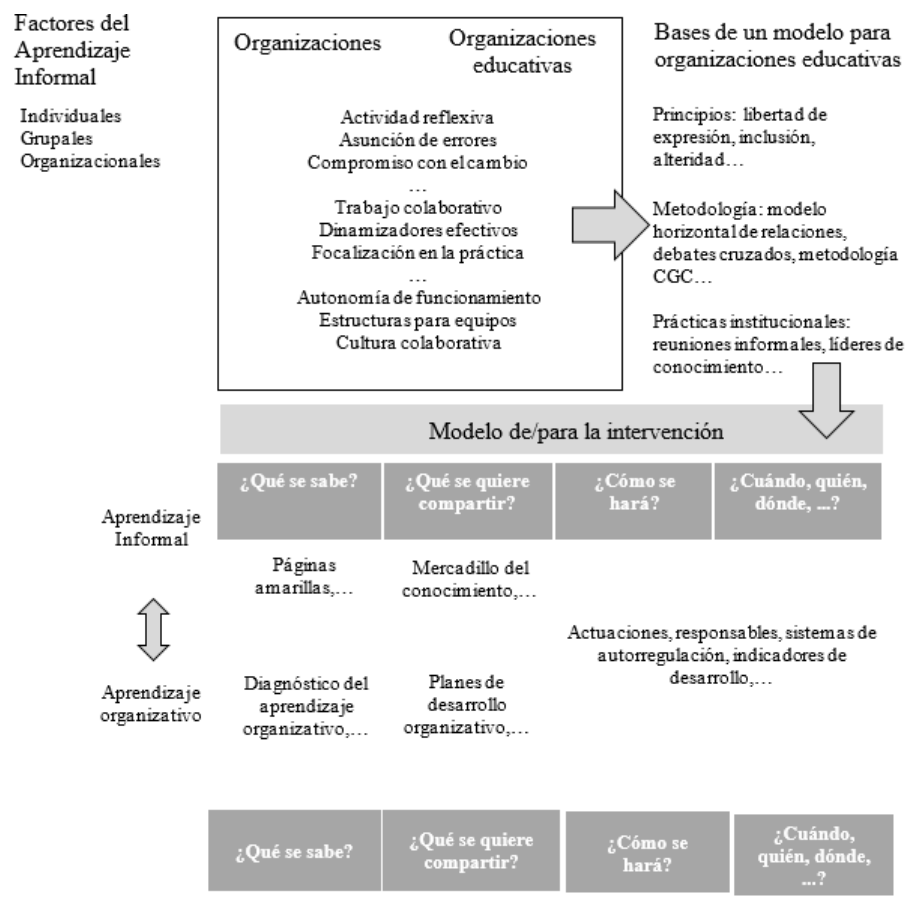

Figura 2 - Propuesta pedagógica para potenciar el aprendizaje informal.

Fuente: Base de datos de investigación.

Elaboración propia.

1 Por ejemplo: http://pagines.uab.cat/pbe/ca. Acceso en: 18 mayo 2020.

2 Por ejemplo, al red de competencias básicas de Catalunya: http://xtec.gencat.cat/ca/ curriculum/xarxacb. Acceso en: 18 mayo 2020. 
progresiva de espacios abiertos en los centros educativos y en la red que invitan a profesionales e instituciones a participar en retos educativos o la progresiva concepción de las salas de profesores como espacios para compartir y promover el aprendizaje informal.

Los factores individuales, grupales y organizacionales identificados y analizados pueden constituir la base para definir la propuesta propia de un centro educativo. Como toda propuesta pedagógica, debería partir de unos principios, que podrían ser los de libertad de expresión, relaciones horizontales, inclusión, alteridad u otros relacionados con una comunicación efectiva e integradora. Asimismo, tendría que especificar una metodología de trabajo (que podría ser la propia de los grupos de creación y gestión del conocimiento colectivo) y las prácticas institucionales que se estiman más adecuadas (reuniones informales sobre temáticas, comisiones ad hoc, estructuras existentes, etc.). Además, se hace necesaria su contextualización a tenor de la realidad de cada institución educativa, de sus características y tipología, así como la del profesorado a quien se dirige (etapa educativa, experiencia, vinculación contractual, etc.), puesto que son elementos influyentes en su aplicación.

La propuesta pedagógica establecida debería no solo ser explicativa sino, y especialmente, estar pensada para la intervención. Siendo así, su concreción en un proyecto de trabajo institucional ha de traducirse en una intervención que albergue actuaciones integradas de carácter individual e institucional. Las actuaciones se pueden dirigir tanto a la creación de un clima de confianza institucional y de unas condiciones de partida adecuadas, como estar orientadas por los procesos propios de intervención. Las estrategias para utilizar en cada uno de los casos pueden ser muy diversas y coincidentes con algunas de las propuestas por Gairín (2016). Este trabajo fundamenta y presenta una guía para la autoevaluación de las comunidades de práctica profesional, proporcionando más de 40 estrategias e instrumentos para la intervención y ejemplos de prácticas exitosas. Otras propuestas pueden analizarse a partir de la documentación publicada de los Congresos Internacionales EDO (Gairín, 2010, 2012, 2016; Gairín y Barrera, 2014; Gairín y Mercader, 2018).

Lo distintivo aquí es que el aprendizaje está en manos de las personas y que el aprendizaje formal tiene un nuevo aliado. Implica, además, replantear el uso actual del conocimiento en las organizaciones: atender a los conocimientos cuando se necesitan, valorar más el conocimiento que se pide que no el solicitado, acomodar el conocimiento al usuario, adaptación del conocimiento antes de que sea adoptado, eficacia del conocimiento personalizado o rentabilidad de las conexiones por encima de los contenidos. Promoverlo en los centros educativos cuenta, al respecto, con diversas estrategias metodológicas que se pueden emplear para mejorar los aprendizajes informales (por ejemplo: práctica reflexiva, supervisión contextual, formación por proyectos aplicados, work shadowing o guía) y los aprendizajes organizativos (por ejemplo: knowledge café, after-action review, estudio de caso, intercambio de conocimientos, knowledge marketplace y peer assist). Ambos tipos cobran sentido ante los propósitos establecidos y lo que cabe plantear es su adaptación y aprovechamiento desde la integración con otras propuestas más formales. 


\section{POSIBILIDADES Y LIMITACIONES A MODO DE CONCLUSIÓN}

El aprendizaje informal siempre se da y se dará. Lo importante en el contexto actual es identificar vías para potenciar los intercambios entre los profesionales y que sirvan tanto para su desarrollo profesional como para el desarrollo de la organización.

Las organizaciones tienen que estimular el aprendizaje informal, dando oportunidades para generar nuevas ideas y nuevos conocimientos, impulsar procesos de cambio y de innovación educativa, mejorar las respuestas institucionales hacia el aprendizaje y la formación, proporcionar una mejor calidad de los servicios que se ofrecen o, incluso, generar beneficios (Cross, 2006; López y Marcelo, 2003).

Las organizaciones pueden desarrollar el aprendizaje informal a través de diferentes tipos de actividades que, principalmente, reparen en (López y Marcelo, 2003):

- Visualización. Las organizaciones facilitan y proporcionan recursos e instrumentos que permitan a sus miembros comunicarse de una manera más ágil y efectiva.

- Conversaciones físicas y/o virtuales, como uno de los elementos principales para el aprendizaje, el descubrimiento y proporcionar e intercambiar ideas, propuestas y, en definitiva, conocimiento.

- Conexiones. Las organizaciones deben promoverlas a través de diferentes canales, y garantizar que sean comprensibles, analizadas y apoyadas para facilitar el aprendizaje individual y colectivo.

La identificación y el desarrollo del aprendizaje informal permiten hacer conscientes a las personas de sus avances, revitalizan y canalizan sus conocimientos, aprovechando los aprendizajes de cada una de las personas y generando aprendizajes colectivos en la línea del aprendizaje organizativo. Algunas otras posibilidades a las que se asocia tienen que ver con la capacidad autoformativa (autonomía), la orientación práctica y aplicativa a las actividades formativas y profesionales vinculadas al puesto de trabajo, la demostración de habilidades prácticas por parte de los profesionales implicados y las oportunidades de empleabilidad al ampliar sus competencias profesionales.

Por otra parte, la consideración de los aprendizajes informales se alinea con los más modernos procedimientos de gestión de las personas y de los recursos humanos en las organizaciones. Es más, se hace referencia a humanos con recursos al comprender que el centro son los profesionales y su capital intelectual la base sobre la que se asienta el progreso de las organizaciones.

Lo relevante de este aprendizaje y del generado en el marco de la educación no formal están modificando los tradicionales modelos y estructuras de formación en las organizaciones (Rodríguez y Gairín, 2015). Así, actualmente es posible pedir el acceso a la universidad sin titulación previa y son abundantes los comités que tratan de validar los conocimientos que los profesionales han podido adquirir por distintos medios (por ejemplo, las comisiones de validación de la experiencia profesional).

De hecho, la certificación de los aprendizajes informales es uno de los retos más importantes de la relación entre formación y trabajo. Fundamentalmente, porque esta tiene que ver con el proceso de validar los conocimientos, el saber-hacer 
y/o las destrezas y competencias adquiridas por las personas mediante el aprendizaje informal y a partir de un procedimiento de evaluación estandarizado (MECU,2019). Una certificación adecuada de los aprendizajes informales tiene diversas ventajas:

- Para las personas: conseguir una titulación que acredite su cualificación profesional en base a su formación y experiencia laboral; adquirir los requisitos de acceso (titulación) para acceder a programas formativos formales; y aumentar sus posibilidades de mejora en la carrera y en el desarrollo profesional.

- Para las organizaciones (INCUAL, 2011): mejorar la productividad de la organización, al contar con profesionales eficientes que mejoran continuamente su cualificación profesional, siendo más capaces de responder adecuadamente a los constantes cambios que tienen lugar; $y$ disponer de profesionales cualificados, a través del reconocimiento de sus competencias profesionales y mediante el establecimiento de procesos que les permitan actualizarlas y mejorarlas permanentemente.

Desde distintos procedimientos (evaluar el aprendizaje experiencial, resultados de aprendizaje y competencias aplicadas, valoración de las propias competencias a partir de la reflexión crítica, etc.), se trata de verificar el grado de vinculación de los nuevos conocimientos con el desarrollo profesional. El logro de procesos de certificación fiables exige colaboración y trabajo conjunto entre organizaciones, universidades, instituciones formativas u otras instancias para que la formación mediante el aprendizaje informal suponga un auténtico valor añadido para las personas y para las organizaciones. Por el contrario, la cualificación se convierte en un problema si no existe un reconocimiento social y personal, institucional y profesional, de los resultados del aprendizaje.

En cualquier caso, la relativa novedad del proceso y la clara competencia que se genera respecto a los sistemas formales de formación, hacen que aún sea tortuoso avanzar. Coincidimos, por consiguiente, con Machado y Pohl (2004) cuando aluden a algunas limitaciones. A nivel personal, los distintos procesos se encuentran determinados por los propios individuos (las personas deciden qué aprender, en qué momento y a qué ritmo), y las dificultades surgen porque no siempre coinciden con los objetivos de la organización. También, están limitados, por otra parte, a un contexto determinado y se adecúan a las necesidades de la situación donde se producen, pero en ocasiones pueden restringir el aprendizaje colaborativo.

Otras limitaciones apuntan a la falta de reconocimiento del aprendizaje informal en las organizaciones, su desaprovechamiento y a la dificultad de localizar metodologías fiables para identificarlo y validarlo. En el caso de los centros educativos, se añade la dificultad de moverse en un campo altamente regulado normativamente en lo que respecta al desarrollo profesional (sistemas de acceso, permanencia y promoción de la profesión docente) y altamente supervisado por los movimientos sindicales y sociales. La propuesta pedagógica planteada en esta contribución queda sujeta, en esta perspectiva, a su aplicación en un futuro próximo y a la contrastación de su potencial práctico al servicio de la mejora de los centros 
educativos y del profesorado y, en definitiva, del desarrollo profesional y organizacional desde el aprendizaje informal.

Podemos concluir que el aprendizaje informal se da en distintos niveles: individual, grupal y organizacional. La comprensión plena de este aprendizaje requiere observar la manera como el aprendizaje individual se genera y contribuye al aprendizaje colectivo y viceversa (Marsick, 1991). De lo que no hay duda es que los centros educativos pueden crear y reflejar condiciones que faciliten o impidan el aprendizaje informal.

$\mathrm{El}$ aprendizaje informal es una muy buena oportunidad tanto para el desarrollo profesional del profesorado (Wilson,2017), como para el desarrollo organizacional de los centros educativos. De hecho, el desarrollo profesional del profesorado queda reforzado cuando este gestiona su formación de forma personalizada, cuando tiene protagonismo en las actividades informales y cuando selecciona aquellas que mejor se adaptan a sus necesidades (González-Sanmamed et al., 2019). Asimismo, las organizaciones se pueden desarrollar estableciendo espacios para el aprendizaje informal, distendidos y donde las personas puedan reunirse libremente cuando precisen encontrar soluciones a problemas o prestarse ayuda mutua compartiendo los conocimientos y las experiencias (De La Cruz, 2017).

Sea como fuere, lo que no puede esperarse es lograr el desarrollo profesional del profesorado y el desarrollo organizacional de los centros educativos a través del aprendizaje informal de una manera inmediata. Más bien será algo progresivo y requerirá de compromiso y esfuerzo para aplicar propuestas como las planteadas y conocer su utilidad y viabilidad contextual.

\section{REFERENCIAS}

AECA - Asociación Española de Contabilidad y Administración de Empresas. Aprendizaje organizativo. Madrid: Asociación Española de Contabilidad y Administración de Empresas, 2011.

ARAGAY,X. et al. Preparemos el terreno. 35 claves para propiciar el cambio educativo. Barcelona: Jesuites Educació, 2015. Cuaderno 2. Disponible en: http://h2020.fje.edu/ wp-content/uploads/2016/11/Q2-CAST.pdf. Acceso en: 26 enero 2018.

ARAGAY, X. Reimaginando la educación: 21 claves para transformar la escuela. Barcelona: Paidós, 2017.

ARETS, J. 5 myths about 70:20:10. London: 70:20:10 Institute, 2017. Disponible en: https://702010institute.com/5-myths-702010/. Acceso en: 2 sept. 2018.

ARGYRIS, C.; SCHÖN,D. A. Organizational learning: a theory of action perspective. Addison-Wesley: Reading Ma, 1978.

ARMENGOL, C. El trabajo en equipo en los centros educativos. Barcelona: Cisspraxis, 2002.

AUBREY, R.; COHEN, P. M. La organización en aprendizaje permanente. Estrategias prácticas para ganar ventajas competitivas. Bilbao: Ediciones Deusto, 1995. BAIGORRI, J.; MARTÍNEZ, P.; MONTERRUBIO, E. El reconocimiento oficial del saber profesional obtenido por la experiencia. Hacia la convergencia de políticas 
sociales en Europa. Revista Europea de Formación Profesional, Luxemburgo, n. 37, p. 36-54, 2006.

BARRERA-COROMINAS, A. La transferencia de los aprendizajes adquiridos en comunidades de práctica en la administración pública. 2015. Tese (Doutorado en Educación) - Universitat Autònoma de Barcelona, Barcelona, 2015.

BOLÍVAR, A. Los centros educativos como organizaciones que aprenden. Madrid: La Muralla, 2000.

BOLÍVAR, A. La autoevaluación en la construcción de capacidades de mejora de la escuela como comunidad de aprendizaje profesional. Revista Portuguesa de Investigação Educacional, Porto, v. 14, p. 9-40, 2014. https://doi.org/10.13140/ RG.2.1.1044.7849

BONALS, J. La práctica del trabajo en equipo del profesorado. Barcelona: Graó, 2013. BROWN, J. S.; DUGUID, P. The social life of information. Cambridge: Harvard Business School Press, 2000.

CARMELO, C.; GARCÍA, J.; SOUSA, E. Facilitadores de los procesos de compartir conocimiento y su influencia sobre la innovación. Cuadernos de Economía y Dirección de la Empresa, España, v. 13, n. 42, p. 113-150, mar. 2010. https://doi.org/10.1016/ S1138-5758(10)70005-0

COBO, C.; MORAVEC, J.W.Aprendizaje invisible. Hacia una nueva ecología de la educación. Barcelona: Universitat de Barcelona, 2011.

COMAS, A.; PIQUÉ, B. Estratègies de práctica reflexiva en la formació inicial de mestres d'educació infantil. Barcelona: Graó, 2010.

CROSS, J. Informal learning: rediscovering the natural pathways that inspire innovation and performance. San Francisco: Pfeiffer \& Company, 2006.

CROSS, J. Informal learning: rediscovering the natural pathways that inspire innovation and performance. San Francisco: Pfeiffer \& Company, 2007.

CROSS, J. El aprendizaje informal hoy en día. In: GAIRÍN,J. (ed.). Nuevas estrategias formativas para las organizaciones. Madrid: Wolters Kluwer, 2010. p. 83- 102.

CROSSAN, M.; LANE, H.; WHITE, R. An organizational learning framework: From intuition to institution, Academy of Management. The Academy of Management Review, United States of America, v. 24, n. 3, p. 522-537, jul. 1999. https://doi. org/10.2307/259140

CUADROS, R. Aprendizaje informal y construcción de PLN vía twitter. Un estudio de caso. EDUTEC - Revista Electrónica de Tecnología Educativa, España, n. 51, p. 1-18, 2015. https://doi.org/10.21556/edutec.2015.51.53

DE LA CRUZ, M. E. Aprendizaje en las organizaciones: ¿formal o informal?. NUBE Didáctica, 2017. Disponible en: https://nubedidactica.com/aprendizaje-enlas-organizaciones-formal-o-informal/. Acceso en: 9 jun. 2019.

DEWEY,J. Cómo pensamos. Cognición y desarrollo humano. Barcelona: Paidós, 2010. DOMINGO, A.; GÓMEZ,M. V. La práctica reflexiva: bases, modelos e instrumentos. Madrid: Narcea, 2014. 
DOMÍNGUEZ, G. Vivir la escuela. Desde una práctica reflexiva, crítica e investigadora. Madrid: Ediciones de la Torre, 2002.

ERAUT, M. Informal learning in the workplace. Studies in Continuing Education, United Kingdom, v. 26, n. 2, p. 247-273, jul. 2004. https://doi. org/10.1080/158037042000225245

ERAUT, M. How professionals learn through work. United Kingdom: University of Surrey, 2008. Disponible en: http://goo.g1/2SsJdB. Acceso en: 10 enero 2018.

ESCARABAJAL, A. Comunidades interculturales y un trabajo colaborativo para una sociedad inclusiva. Madrid: Narcea, 2015.

ESCUDERO, J. M.; CUTANDA, M. T.; TRILLO, J. F. Aprendizaje docente y desarrollo profesional del profesorado. Profesorado - Revista de Currículum y Formación del Profesorado, Granada, v. 21, n. 3, p. 83-102, 2017.

FALK, J.; DIERKING, L. (eds.). Public institutions for personal learning: establishing a research agenda. Washington, DC: American Association of Museums, 1995.

FERNÁNDEZ, M. El aprendizaje informal en comunidades de práctica virtuales en la administración pública: evaluación y acreditación. 2014. Tese (Doutorado) Universitat Autònoma de Barcelona, Barcelona, 2014.

GAIRÍN, J. (ed.). Nuevas estrategias formativas para las organizaciones. Barcelona: Wolters Kluwer, 2010.

GAIRÍN, J. (ed.). Gestión del conocimiento y desarrollo organizativo: formación y formación corporativa. Madrid: Wolters Kluwer, 2012.

GAIRÍN, J. (ed.). Las comunidades de práctica profesional. Creación, desarrollo y evaluación. Barcelona: Wolters Kluwer, 2015.

GAIRÍN, J. (ed.). Aprendizaje situado y aprendizaje conectado: implicaciones para el trabajo. Madrid: Wolters Kluwer, 2016.

GAIRÍN, J.; BARRERA, A. (eds.). Organizaciones que aprenden y que generan conocimiento. Madrid: Wolters Kluwer, 2014.

GAIRÍN, J.; MERCADER, C. (eds.). Liderazgo y gestión del talento en las organizaciones. Barcelona: Wolters Kluwer, 2018.

GAIRÍN, J.; SUÁREZ, C. I.; RODRÍGUEZ-GÓMEZ, D. et al. ICEDU-Análisis de los procesos de aprendizaje organizativo e informal en los centros educativos. Validación de propuestas tecnológicas para el desarrollo institucional y profesional docente. Barcelona: Ministerio de Economía y Competitividad; Equipo de Desarrollo Organizacional, 2017.

GALINDO, A.C. Aprendizaje informal desde la cultura participativa: Aristas edupunk como elementos de posibilidad para el desarrollo social. IXAYA - Revista Universitaria de Desarrollo Social, Guadalajara, n. 5, p. 101-120, 2013.

GARCÍA-VALCÁRCEL, A. (coord.). Proyectos de trabajo colaborativo con TIC. Madrid: Síntesis, 2015.

GASALLA, J. M.; NAVARRO, L. Confianza. La clave para el éxito personal y empresarial. Barcelona: Empresa Activa, 2008. 
GONZÁLEZ, J. C.; GUTIÉRREZ, M. Formación innovadora. Nuevos modelos para la formación en la Administración pública. Madrid: INAP, 2013.

GONZÁLEZ-ISASI, R. M. et al. Colaboración en comunidades de práctica para el desarrollo profesional del profesor. Revista de Medios y Educación, Sevilla, n. 42, p. 103-113, enero 2013.

GONZÁLEZ-SANMAMED, M. et al. Aprendizaje informal y desarrollo profesional: análisis de las ecologías de aprendizaje del profesorado de educación infantil. EDUTEC - Revista Electrónica de Tecnología Educativa, España, n. 68, p. 70-81, jun. 2019. https://doi.org/10.21556/edutec.2019.68.1305

INCUAL - Instituto Nacional de las Cualificaciones. Madrid: INCUAL, 2011. Disponible en: http://www.educacion.gob.es/educa/incual/ice_incual.html. Acceso en: 20ene. 2018.

JISC - Joint Information Systems Committee. Effective assessment on digital age. Bristol: JISC, 2010. Disponible en: https://www.jisc.ac.uk/podcasts/ podcastpress-release-effective-assessment-in-a-digital-age-06-sep-2010. Acceso en: 2 jul. 2020.

JENNINGS, C. Trabajar de forma más inteligente utilizando el marco 70/20/10. In: GAIRÍN, J. (ed.). Gestión del conocimiento y desarrollo organizativo. Barcelona: Wolters Kluwer, 2012. p. 53-93.

KOLB, D. A. Experiential learning: experience as the source of learning and development. Englewood Cliffs, NJ: Prentice Hall, 1984.

KRICHESKY, G. J.; MURILLO, J. F. La colaboración docente como factor de aprendizaje y promotor de mejora. Un estudio de casos. Educación XXI, España,v. 21, n. 1, p. 135-156. 2018. https://doi.org/10.5944/educxx1.20181

LAVIE, J. M. El trabajo colaborativo del profesorado. Salamanca: Comunicación Social Ediciones y Publicaciones, 2011.

LLP - Lifelong Learning Programme. Reconocimiento del aprendizaje informal y no formal. Directrices y materiales para los representantes sindicales en la empresa. Unión Europea: IFES, 2010.

LÓPEZ,A. 14 ideas clave. El trabajo en equipo del profesorado. Barcelona: Graó, 2007. LÓPEZ, J.; MARCELO, C. El aprendizaje informal y su impacto sobre el desarrollo organizativo. In: GAIRÍN, J.; ARMENGOL, C. (eds.). Estrategias de formación para el cambio organizacional. Barcelona: Praxis, 2003. p. 477-486.

LÓPEZ-RODRÍGUEZ, F. et al. Dinámicas colaborativas en el trabajo del profesorado: el paso del yo al nosotros. Barcelona: Graó, 2002.

MACHADO,J.; POHL, A. Los dilemas del reconocimiento del aprendizaje informal. Revista de Estudios de Juventud, España, n. 65, p. 83-98, 2004.

MARSICK, V.J. Aprendizaje en el centro de trabajo: el caso de la reflexividad y de la reflexividad crítica. Revista de Educación, Madrid, n. 294, art. 7, p. 141-154, 1991.

MARSICK, V.J.; WATKINS, K. Informal and incidental learning in the workplace. Nueva York: Routledge, 1990. 
MARTÍNEZ,E. F.La colaboración entre profesores como modelo de apoyo y desarrollo docente. Politikon, 28 mar. 2017. Disponible en: https://politikon.es/2017/03/28/ la-colaboracion-entre-profesores-como-modelo-de-apoyo-y-desarrollo-docente/\#. Acceso en: 20 enero 2018.

MARTÍNEZ, X. El model esgotat de secundària en l'escola conservadora pública i l'horitzó dels jesuïtes. E1 Diari de l'Educació, Barcelona, 9 mar. 2015. Disponible en: http://diarieducacio.cat/el-model-esgotat-de-secundaria-en-lescola-conservadorapublica-lhoritzo-dels-jesuites/. Acceso en: 20 enero 2018.

MARTÍNEZ, J.; MUÑOZ, J. L. Aprender en las organizaciones de la era digital. Alternativas desde la formación y para la transformación. Barcelona: Ediciones UOC, 2018.

MARTÍNEZ, J.; MUÑOZ, J. L.; FALIVENE, G. M. Formación y aprendizaje en la administración pública: el modelo 70/20/10. Revista Vasca de Gestión de Personas y Organizaciones Públicas, España, n. 13, p. 44-57, 2017.

MARTÍNEZ-ALDANONDO, J. La creación y gestión del conocimiento. In: GAIRÍN, J. (ed.). Nuevas estrategias formativas para las organizaciones. Madrid: Wolters Kluwer, 2010. p. 69- 82.

MARTÍNEZ-ALDANANDO,J.Tres hábitos para gestionar conocimiento. Catenaria -Gétion del Conocimiento, n. 118, 2016. Disponible en: http://www.catenaria.cl/km/ newsletter/newsletter_118.htm. Acceso en: 18 oct. 2017.

MARTÍNEZ MARÍN, J. Nuevas estrategias formativas para las administraciones públicas. In: GAIRÍN, J. (ed.). Nuevas estrategias formativas para las organizaciones. Madrid: Wolters Kluwer, 2010. p. 103-119.

MECU - Marco Español de Cualificaciones. Glosario. Madrid: Ministerio de Educación y Formación Profesional, 2019. Disponible en: https://www.educacionyfp. gob.es/educacion/mc/mecu/preguntas-frecuentes-glosario.html. Acceso en: 30 jun. 2019.

MEJÍA, R. Tendencias actuales en la investigación del aprendizaje informal. Sinéctica, México, n. 26, p. 1-8, 2005.

NICOL, D. Re-engineering assessment practices in higher education. Glasgow: University of Strathclyde Glasgow, 2010. Disponible en: www.reap.ac.uk. Acceso en: enero 2017.

OLMOS, J. J. Una propuesta para la gestión y evaluación del trabajo cooperativo. Alcoy: Editorial Academia Española, 2012.

OROZCO, C. E. ¿Medir lo inmensurable? Evaluar el aprendizaje en ambientes informales. Sinéctica, México, n. 26, p. 94-97, 2005.

PADILLA, M. T.; GIL, J. La evaluación orientada al aprendizaje en la educación superior: condiciones y estrategias para su aplicación en la docencia universitaria. Revista Española de Pedagogía, España, n. 241, p. 467-486, 2008.

PEARN, M.; RODERICK, C.; MULRRONEY, C. Learning organizations in practice. London: McGraw-Hill, 1995. 
PERRENOUD, P. Desarrollar la práctica reflexiva en el oficio de enseñar. Barcelona: Graó, 2004.

PRIETO, I. M. Gestión del conocimiento para el desarrollo de la capacidad de aprendizaje en las organizaciones. Valladolid: Secretariado de Publicaciones e Intercambio Editorial; Universidad de Valladolid, 2005.

QUINTO, B.; FENERO, M. J. Educar en el 0-3. La práctica reflexiva en los nidi d'infanzia. Barcelona: Graó, 2016.

RAMÍREZ,M.S.; GARCÍA,F.J. Co-creación e innovación abierta: revisión sistemática de literatura. Comunicar - Revista Científica de Comunicación y Educación, España, v. XXVI, n. 54, p. 9-18, 2018. https://doi.org/10.3916/c54-2018-01

RETORTILLO, A. La evaluación, reconocimiento y acreditación de los aprendizajes no formales e informales en el ámbito universitario: elementos para el debate. REIFOP - Revista Electrónica Interuniversitaria de Formación del Profesorado, España, v. 14, n. 1, p. 218-226, 2011.

RIERA, C. Aprenentatge entre iguals i aprenentatge informal a l'organització. Plantejament d'un nou itinerari d'investigació per a l'aprenentatge organitzatiu. 2013. Tese (Doutorado) - Universitat Autònoma de Barcelona, Barcelona, 2013.

RODRÍGUEZ, D.; GAIRÍN, J. Innovación, aprendizaje organizativo y gestión del conocimiento en las instituciones educativas. Educación, Lima, n. 46, p. 73-90, 2015.

ROGET, D. Práctica reflexiva para docentes. De la reflexión ocasional a la reflexión metodológica. Saarbrücken: Publicia, 2013.

SALINAS, J. Acceso a la información y aprendizaje informal en internet. Comunicar - Revista Científica de Comunicación y Educación, España, v. XI, n. 21, p. 31-38, 2003. https://doi.org/10.3916/C21-2003-05

SÁNCHEZ-VALLE, M. Gestión de la participación en las organizaciones: un modelo de comunicación empática. Estudio realizado en la facultad de comunicación de la Universidad Pontificia de Salamanca. 2003. Tese (Doutorado) - Universidad Pontificia de Salamanca, Salamanca, 2003.

SANGRÀ, A.; WHEELER, S. Nuevas formas de aprendizaje informales: ¿ $\mathrm{O}$ estamos formalizando lo informal?. RUSC-Revista de Universidad y Sociedad del Conocimiento, España, v. 10, n. 1, p. 107-115, 2013. http://dx.doi.org/10.7238/rusc.v10i1.1689

SANZ, S. Comunidades de práctica virtuales: acceso y uso de contenidos. RUSC Revista de Universidad y Sociedad del Conocimiento, España, v. 2, n. 2, p. 26-35, 2002. SCHÖN, D. A. El profesional reflexivo. Cómo piensan los profesionales cuando actúan. Barcelona: Paidós, 1998.

SCOTT, S.; FERGUSON, O. New perspectives on 70:20:10. A good practice research paper. [S.l.]: GoodPractice, 2016. Disponible en: http://ww.w.cedma-europe.org/ newsletter\%20articles/misc/New\%20Perspectives\%20on\%2070-20-10\%20(Nov\%20 14).pdf. Acceso en: 19 mayo 2020.

SENGE, P. M. La quinta disciplina. El arte y la práctica de la organización abierta al aprendizaje. Barcelona: Ediciones Granica, 1992. 
SERRANO, G.; DALMAU, O. Agentes facilitadores del aprendizaje y el conocimiento en las organizaciones. Equipos \& Talento, España, p. 18-19, 2010.

TRILLA, J. La educación informal. Barcelona: PPU, 1987.

TRILLO, F. et al.El desarrollo profesional y la colaboración docente: un análisis situado en el contexto español de las tensiones y fracturas entre la teoría y la práctica. Archivos Analíticos de Políticas Educativas, Estados Unidos, v. 25, n. 117, p. 1-32, 2017. https:// doi.org/10.14507/epaa.25.3316

UNESCO - Organización de las Naciones Unidas para la Educación, la Ciencia y la Cultura. Directrices de la UNESCO para el reconocimiento, validación y acreditación de los resultados del aprendizaje no formal e informal. Hamburgo: UNESCO, 2012. WATKINS, K.; MARSICK, V.J. Sculpting the learning organization. San Francisco: Jossey-Bass, 1993.

WILSON, D. Los aprendizajes informales son la mejor oportunidad para el crecimiento. Entrevista a Daniel Wilson, investigador principal de Project Zero, equipo de investigación educativa de la Harvard Graduate School of Economics. [Entrevista cedida a] Pau Rodriguez. El Diario de la Educación, 3 abr. 2017. Entrevista. Disponible en: https://eldiariodelaeducacion.com/blog/2017/04/03/los-aprendizajes-informales-sonla-mejor-oportunidad-para-el-crecimiento/. Acceso en: jul. 2019.

\section{SOBRE LOS AUTORES}

Joaquín Gairín Sallán es doctor en filosofía y letras por la Universitat Autònoma de Barcelona (España). Profesor de la misma institución.

E-mail: joaquin.gairin@uab.cat

José Luís Muñoz Moreno es doctor en educación por la Universitat Autònoma de Barcelona (España). Profesor de la misma institución.

E-mail: joseluis.munoz@uab.cat

Patricia Silva García es doctora en psicología de la pedagogía por la University of Lleida (España). Profesora de la misma institución.

E-mail: silva@pip.udl.cat

Cecilia Inés SuÁrez es doctora en educación por la Universitat Autònoma de Barcelona (España). Profesora de la misma institución. E-mail: ceciliaines.suarez@uab.cat

Recibido el 04 de febrero de 2019

Aprobado el 10 de febrero de 2020 